\title{
The Contribution of Real-Time Mirror Reflections of Motor Actions on Virtual Body Ownership in an Immersive Virtual Environment
}

\author{
Mar González-Franco ${ }^{1}$ \\ ${ }^{1}$ EVENT Lab \\ Facultat de Psicologia \\ Universitat de Barcelona, \\ Spain
}

\author{
Daniel Pérez-Marcos ${ }^{1,2}$ \\ ${ }^{2}$ Institut d'Investigacions \\ Biomèdiques August $\mathrm{Pi}$ i \\ Sunyer (IDIBAPS), \\ Barcelona, Spain
}

\author{
Bernhard Spanlang ${ }^{1,3}$ \\ ${ }^{3}$ Departament de LSI \\ Universitat Politècnica de \\ Catalunya, Spain
}

\author{
Mel Slater ${ }^{1,4,5}$ \\ ${ }^{4}$ Institució Catalana de \\ Recerca i Estudis Avançats \\ (ICREA), Spain \\ ${ }^{5}$ Department of Computer \\ Science, University College \\ London, UK
}

\begin{abstract}
This paper reports an experiment that investigated people's body ownership of an avatar that was observed in a virtual mirror. Twenty subjects were recruited in a within-groups study where 10 first experienced a virtual character that synchronously reflected their upper-body movements as seen in a virtual mirror, and then an asynchronous condition where the mirror avatar displayed prerecorded actions, unrelated to those of the participant. The other 10 subjects experienced the conditions in the opposite order. In both conditions the participant could carry out actions that led to elevation above ground level, as seen from their first person perspective and correspondingly in the mirror. A rotating virtual fan eventually descended to $2 \mathrm{~m}$ above the ground. The hypothesis was that synchronous mirror reflection would result in higher subjective sense of ownership. A questionnaire analysis showed that the body ownership illusion was significantly greater for the synchronous than asynchronous condition. Additionally participants in the synchronous condition avoided collision with the descending fan significantly more often than those in the asynchronous condition. The results of this experiment are put into context within similar experiments on multisensory correlation and body ownership within cognitive neuroscience.
\end{abstract}

KEYWORDS: rubber hand illusion, body ownership, agency, virtual reality

INDEX TeRMS: J.4 [Social and Behavioral Sciences]: Psychology; I.3.6 [Computer Graphics]: Methodology and TechniquesInteraction techniques; I.3.7 [Computer Graphics]: ThreeDimensional Graphics and Realism-Virtual Reality.

\section{INTRODUCTION}

How do we know what is and what is not part of our own body?

e-mail: margonzalez@ub.edu

e-mail: dperez1@clinic.ub.es

e-mail: bspanlang@ub.edu

e-mail: melslater@ub.edu

IEEE Virtual Reality 2010

20 - 24 March, Waltham, Massachusetts, USA

978-1-4244-6238-4/10/\$26.00 @2010 IEEE
This issue of body ownership has received increasing scientific attention in recent years. Current findings suggest that visuotactile and visuomotor correlations play an important role in how the brain decides ownership. By manipulating these correlations, it is possible to generate illusions that alien or even objects in virtual reality can be felt as part of one's own body. The most wellknown example of this is the rubber hand illusion [2]. In this case, the real arm of a participant is hidden from their view, and a rubber arm placed in a plausible position on a table in front of them. The experimenter synchronously taps and strokes the real hand of the person and the rubber hand. After a few seconds of stimulation most people have the illusion that the rubber hand is their own hand. This is demonstrated both subjectively, through the use of a questionnaire, and behaviorally using a proprioceptive drift measurement. This is the distance between the felt position of their hand as blindly pointed out before the stimulation and the felt position blindly pointed out afterwards. When the visual and tactile stimulation are synchronous the proprioceptive drift is typically significantly different from zero, and towards the position of the rubber hand. When the stimulation is asynchronous, both the questionnaire scores and the drift scores do not indicate the change in ownership illusion. Physiological response to threat towards the rubber hand has also been used as an objective measure of ownership $[1,5]$. The rubber hand illusion has also been shown to work in virtual reality, where both the real and a computer-generated 3D arm were synchronously stimulated with real (tactile) touches and virtual (visual) touches, respectively [14].

This method has also been extended towards ownership of the whole body using similar techniques. This employs tactile stimulation to the person's (unseen) real body and corresponding visual stimulation to a displaced video image of their body as seen through a head-mounted display (HMD). When the visuotactile stimulation is synchronous an out-of-the-body illusion occurs, as if the participants were in the displaced body, as evidenced by questionnaire based subjective responses and a behavioral response akin to proprioceptive drift [11] or physiological arousal in response to a threat to the virtual body [6]. A similar idea has been used to generate ownership of a manikin that appears to replace the body of the participant [12]. A recent review of this area of research can be found in [15].

The rubber hand illusion has been extended to visuomotor correlations between the movements of the hidden real hand and 
the rubber hand $[14,15]$. This is related to agency, 'The sense that I am the one who is causing or generating an action' [7]. However, the overwhelming majority of work in this area has concentrated on visuotactile correlations.

Here we concentrate on agency with respect to the body as a whole, exclusively examining the contribution of real-time virtual mirror reflections of upper body movements on body ownership. We compare two conditions - when the movements of the avatar seen in the mirror are synchronous with those of the participant, and an asynchronous condition, where the movements of the avatar were based on pre-recorded human movements similar to those that were required by the experiment. We employed a subjective response measure based on a questionnaire, and a behavioral measure based on the response of the participant to a threat. We expected that in the synchronous condition there would be greater subjective sense of body ownership, and a behavioral response that indicated the avoidance of harm to the reflected virtual body.
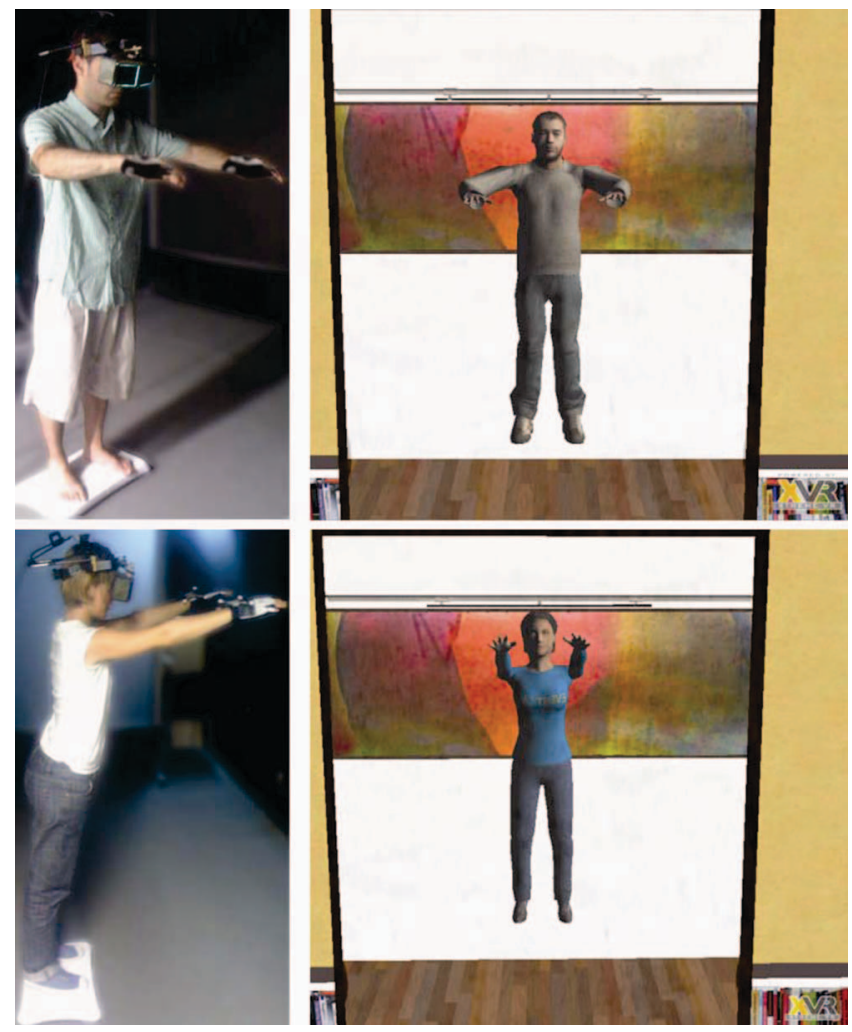

Figure 1. The participant's upper body movements and rotations are reflected in a virtual mirror. When the participant's arms are raised or when leaning forward on a Wii board, their viewpoint and their mirror reflection will elevate.

\section{MATERIALS AND METHODS}

\subsection{Equipment and Software}

The environment was displayed via a Fakespace Labs Wide5 HMD, which has field of view $150^{\circ} \times 88^{\circ}$ with an estimated 1600 $\times 1200$ resolution. The software environment was XVR [3], and the virtual character was displayed using a hardware accelerated avatar library [8]. Inverse kinematics was implemented using the hand position data from infrared tracking (12 camera OptiTrack) and the head position data from an Intersense 900. Elbow and shoulder rotations were calculated from hand position; spine and head rotations were calculated from tracked head rotation and position. Balance data were registered using the Wii Balance Board from Nintendo. Our setup supported the real-time display of the avatar with movements of its upper body reflecting movements of the participant. The resulting real-time avatar movements included 3 degrees of freedom (DOF) in the head; 2 DOF in the spine; and 4 DOF in both arms (Fig. 1).

The scenario consisted of a room of size $7 \times 5 \times 7 \mathrm{~m}^{3}$. The scene contained a mirror and just in front of it there were a chair and a child's toy; behind the mirror there was a library against the wall; on the back wall there was a painting; and on the left wall there was a large window. The participant stood $2 \mathrm{~m}$ from the mirror of size $1.5 \times 3 \mathrm{~m}^{2}$. In the mirror a male or female virtual character was displayed. There were two conditions, one where the avatar faithfully mirrored the motor actions of the participant, where all upper body movements were tracked and reflected as explained in Section 2.1. In the second condition, pre-recorded movements were applied to the avatar, so that the avatar's upper body moved independently of the movements of the participant.

In both the synchronous and asynchronous conditions when the participant leaned forward on the Wii board or raised their arms then they would see their avatar reflection float upwards. The effect of these two methods (arm raising and leaning forward) was additive and contributed equally to the final visual effect. Therefore the participants could elevate their viewpoint in the virtual environment (i.e. virtually levitate) by leaning forward and/or by raising their arms. The maximum height that could be reached was $3.2 \mathrm{~m}$. The only virtual body visible was the one in the mirror reflection.

A rotating virtual fan of $2.5 \mathrm{~m}$ diameter was suspended from the ceiling of the room and located directly above the participant's reflection; it could only be seen in the mirror. Initially it was at a height of $4.5 \mathrm{~m}$. Eventually the rotating fan started to move lower and lower until it reached a height of $2 \mathrm{~m}$, while continuously rotating. A real fan like that would cause harm if it collided with the body of a person. There were no associated auditory cues.

\subsection{Procedures and Experimental Design}

Ten men and ten women were recruited from the campus of University of Barcelona. Their ages ranged between 18 and 31 years. On entering the virtual reality laboratory they were given an information sheet to read that described equipment to be used and the procedures of the experiment. They were advised that they could withdraw at any time without giving reasons, and given an informed consent form to sign.

They were then taken to the area where the experiment was to be carried out, and the operation of the Wii board was explained to them. They were told that they should adopt different upper body postures, including bending all the way forward while keeping their legs straight, and to try to understand the relationship between their movements and what they would see in the virtual reality.

Then they were helped to put on the HMD and at first the environment did not display the mirror reflection. This provided the opportunity to adjust the HMD until it was comfortable, and the visual clarity maximized. The procedures were then explained once again. The participants were asked to close their eyes, and when they opened them again they could see the mirror reflection. Males saw a male virtual character and females a female one.

Participants could now try out various body postures and observe the effects for another 30s. The participants learned that when leaning forward or raising the arms, the body of the avatar in the mirror floated upwards and (only in the synchronous condition) the upper-body movements in the avatar corresponded 
with those of the participant. When the avatar floated above the ground, the participants' viewpoint reflected this so that with respect to the floor of the room, they would always be at the same height as the avatar. After a further 3 minutes of body movement, the rotating fan in the mirror descended over a period of $26 \mathrm{~s}$ until its height (measured with respect to ground) was $2 \mathrm{~m}$. Hence, if the person floated above the ground they would reach a point where they would collide with the fan.

At the end of this period, participants were again asked to close their eyes, and a series of questions was read out to them (next section). Then the experience was repeated for a second time (without the training aspects) but now in the other condition. The overall experimental design was therefore within-groups where each of the 20 participants experienced both synchronous and asynchronous conditions, counterbalanced for order. Moreover, each condition was counterbalanced for gender. All the subjects were paid $5 €$ for their participation. The experiment had been approved by the Comité Ético de Investigación Clínica at Hospital Clinic Barcelona.

\subsection{Questionnaire Responses}

At the end of each of the two experiences the following questions were read out to the participants (in Catalan, Spanish or English depending on the preference of the participant) and they were asked to answer on a scale from 1 (disagree strongly) to 5 (agree strongly) concerning their level of agreement with the following sentences:

mybody: I felt as if the body I saw in the mirror might be my body harmed: I had the feeling that I might be harmed if I collided with the air fan

mymovement: The movements I saw the body in the mirror make seemed to be my movements

otherperson: The body I saw in the mirror was another person

floating: I felt as if my real body were floating above the ground.

The critical questions are mybody and harmed, since these directly indicate the illusion of ownership. Mymovement and otherperson were for consistency checks where participants in the synchronous condition should answer positively to first and negatively to the second, and vice versa for those in the asynchronous condition. Floating is another consistency check question since in both synchronous and asynchronous conditions the body floated above the ground, and we would not expect any significant difference between the conditions.

\subsection{Behavioral Response}

The participants had been encouraged to understand the relationship between their upper-body movements and the corresponding results. They had also been encouraged to float above the ground. The question was how much they would take notice of the rotating fan. Of course, everyone knew that the fan could have no effect whatsoever on themselves. However, a sign of body ownership towards their virtual body as seen in the mirror would be if the participants chose to limit the height to which they floated as the fan descended. The hypothesis was that they would have a greater propensity to stay below the fan in the synchronous compared to the asynchronous condition.

\section{Results}

\subsection{Questionnaire Responses}

We used a repeat-measures Analysis of Variance, where the main factor was Body Movement (synchronous or asynchronous). There were two experimental groups: AS - those that had the asynchronous condition first followed by synchronous and SA - those that had the opposite order. The means and standard errors for each condition and group are shown in Table 1 . The repeatmeasures ANOVA tests for differences between the means of the responses under the conditions of the main factor (synchronous, asynchronous) allowing for differences between the groups (i.e., order effects) and also between subjects, and interactions between these. The $\mathrm{P}$ values in Table 1 give the significance levels for the difference between the means under the ANOVA for each variable. Jarque-Bera tests [10] on the residual errors of each of the fits showed that these all were compatible with normally distributed errors.

The results conform to our expectations - the synchronous condition resulted in greater signs of body ownership than the asynchronous, and the consistency questions also conform to expectation.

Table 1: Means and Standard Errors of the Conditions

\begin{tabular}{|c|c|c|c|c|c|c|}
\hline & & Async & onous & Synch & nous & \\
\hline & Group & Mean & SE & Mean & SE & $\mathrm{P}$ \\
\hline mybody & AS & 2.30 & 0.37 & 4.30 & 0.15 & 0.000 \\
\hline & SA & 1.50 & 0.17 & 3.70 & 0.21 & \\
\hline harmed & $\mathrm{AS}$ & 2.20 & 0.36 & 2.60 & 0.31 & 0.003 \\
\hline & SA & 1.50 & 0.31 & 3.00 & 0.30 & \\
\hline mymovement & $\mathrm{AS}$ & 2.20 & 0.33 & 4.50 & 0.17 & 0.000 \\
\hline & SA & 1.20 & 0.13 & 3.80 & 0.13 & \\
\hline otherperson & $\mathrm{AS}$ & 4.10 & 0.23 & 2.80 & 0.33 & 0.000 \\
\hline & SA & 4.60 & 0.22 & 2.90 & 0.41 & \\
\hline floating & $\mathrm{AS}$ & 3.60 & 0.31 & 3.90 & 0.28 & 0.140 \\
\hline & SA & 2.60 & 0.48 & 3.20 & 0.39 & \\
\hline Proportion of & $\mathrm{AS}$ & 0.47 & 0.07 & 0.64 & 0.08 & 0.031 \\
\hline $\begin{array}{l}\text { times head } \\
\text { below the fan }\end{array}$ & SA & 0.47 & 0.11 & 0.60 & 0.07 & \\
\hline
\end{tabular}

There were no significant interaction effects between Body Movement and group. Only in the case of mybody was there an order effect $(\mathrm{P}=0.01)$. This is wholly accounted for by those in the AS group giving a higher score for both asynchronous and synchronous conditions than those in the SA group.

\subsection{Behavioral Response}

The results support the notion that participants in the synchronous condition tended to stay below the fan more during the period when it was descending. We computed the number of jumps in this period and the maximum height of the avatar in each jump, and therefore the proportion of times that the maximum avatar height was less than the height of the fan. The last two rows of Table 1 give the results. The repeat-measures ANOVA shows a significant difference between the asynchronous and synchronous conditions and no order or interaction effect.

\section{Discussion}

The questionnaire results indicate that there is a subjective illusion of body ownership that is very strong, and there is also clear behavioral evidence supporting this conclusion. To the authors' knowledge this is the first study of the effects of agency on whole body ownership, and the first that employs a virtual mirror. It could be argued that there was some similarity between the two conditions since in both the viewpoint was raised when the participants leaned forward and/or stretched out their arms. However, in the asynchronous condition these leaning forward and arm stretching actions were not mirrored by the avatar reflection, only the viewpoint was raised. Hence, our results 
strongly support the conclusion that it was the upper-body movement mirroring alone that was responsible for the difference between the conditions, since the elevation in viewpoint was the same for both.

The relative importance of agency for body ownership is not as well understood as visual-tactile correlations. In [4] a version of the rubber hand illusion was used, where the rubber hand moved synchronously with the subject, without tactile stimulation. Here the subjective, questionnaire-based illusion was found. In [15] a study was reported where both the subjective questionnaire response and proprioceptive drift was found in a virtual reality study that compared synchronous visual-motor movements of a virtual hand with an asynchronous condition. We suspect that overall agency to be a contributory factor to body ownership but not as decisive as co-location (where the virtual body substitutes the own body) combined with visual-tactile synchrony [16].

\section{Conclusion}

We have reported a study that shows that it is possible to obtain a body ownership illusion of a mirrored virtual body image when there is synchrony between motor actions of the participant and the seen movements of the avatar mirrored image. The only acknowledgement towards the actual appearance of the participants was that males were represented by a male virtual body and females by a female one. It would be interesting to examine the impact on the illusion of likeness of appearance between the actual face and body of the participant with that of the virtual representation.

There are several applications of these results. First, in the area of stroke rehabilitation it is becoming increasingly common to employ a form of virtual reality [9]. Here the patient observes their virtual limb moving with capabilities that may be enhanced compared to their actual possible behavior. We speculate that a body ownership with respect to the virtual body might enhance the effectiveness of such treatment. Moreover, patients are likely not to be in a first person position in relation to their virtual body but see it on an external screen. Although in our experiment we used a HMD, the mirror effect could be achieved also with a projection display. The second application is in the general area of interaction within virtual reality. Overall the greater the sense of ownership over one's virtual representation, the greater the possibility for presence, that is the response to virtual events and situations as if they were real [13]. Finally, such an interface, employing agency as much as possible, is a natural extension of modern gaming interfaces such as the Wii system.

Overall, the area of research that is the intersection between computer science (in particular virtual reality) and cognitive neuroscience with respect to the issue of body ownership, is highly interesting, with likely positive benefits for both disciplines.

\section{ACKNOWLEDGMENTS}

The authors wish to thank Konstantina Kilteni for her help with the experiment and Iason Kastanis for the Wii balance board interface. Financial support from: TV3 Catalunya 'Dolor Crónico' 2006, the EU FET PRESENCCIA project contract Number 27731, the EU project MIMICS, and the ERC project TRAVERSE.

\section{REFERENCES}

[1] K. C. Armel, and V. S. Ramachandran, "Projecting sensations to external objects: evidence from skin conductance response,"
Proceedings of the Royal Society of London Series BBiological Sciences, vol. 270, no. 1523, pp. 1499-1506, 2003.

[2] M. Botvinick, and J. Cohen, "Rubber hands 'feel' touch that eyes see,” Nature, vol. 391, no. 6669, pp. 756-756, 1998.

[3] M. Carrozzino, F. Tecchia, S. Bacinelli et al., "Lowering the development time of multimodal interactive application: the real-life experience of the XVR project." pp. 270-273.

[4] T. Dummer, A. Picot-Annand, T. Neal et al., "Movement and the rubber hand illusion," Perception, vol. 38, no. 2, pp. 271280, 2009.

[5] H. Ehrsson, K. Wiech, N. Weiskopf et al., "Threatening a rubber hand that you feel is yours elicits a cortical anxiety response," Proceedings of the National Academy of Sciences, vol. 104, no. 23, pp. 9828, 2007.

[6] H. H. Ehrsson, "The experimental induction of out-of-body experiences," Science, vol. 317, no. 5841, pp. 1048-1048, Aug 24, 2007.

[7] S. Gallagher, "Philosophical conceptions of the self: Implications for cognitive science," Trends in Cogn. Sci, vol. 4, pp. 14-21, 2000.

[8] M. Gillies, and B. Spanlang, "Comparing and evaluating realtime character engines for virtual environments," PRESENCE Teleoperators and Virtual Environments, vol. in press, 2009.

[9] M. K. Holden, "Virtual environments for motor rehabilitation: review," Cyberpsychology \& behavior, vol. 8, no. 3, pp. 187211, 2005.

[10] C. M. Jarque, and A. K. Bera, "Efficient tests for normality, homoscedasticity and serial independence of regression residuals," Economics Letters, vol. 6, no. 3, pp. 255-259, 1980.

[11] B. Lenggenhager, T. Tadi, T. Metzinger et al., "Video Ergo Sum: Manipulating Bodily Self-Consciousness," Science, vol. 317, no. 5841, pp. 1096, 2007.

[12] V. I. Petkova, and H. H. Ehrsson, "If I Were You: Perceptual Illusion of Body Swapping," PLoS ONE, vol. 3(12): e3832. doi:10.1371/journal.pone.0003832, 2008.

[13] M. V. Sanchez-Vives, and M. Slater, "From Presence to Consciousness through Virtual Reality," Nature Reviews Neuroscience, vol. 6, no. 4, pp. 332-339, 2005.

[14] M. Slater, D. Pérez Marcos, H. H. Ehrsson et al., "Towards a digital body: The virtual arm illusion," Front. Hum. Neurosci., vol. doi:10.3389/neuro.09.006.2008, 2008.

[15] M. Slater, D. Perez-Marcos, H. H. Ehrsson et al., "Inducing Illusory Ownership of a Virtual Body," Frontiers in Neuroscience, vol. 3, no. 2, pp. 214-220, 2009.

[16] M. Slater, B. Spanlang, M. Sanchez-Vives et al., "First person experience of body transfer in virtual reality," PLOS ONE (minor changes), 2010. 